\title{
MicroRNA-214 promotes the EMT process in melanoma by downregulating CADM1 expression
}

\author{
SHU-JUN WANG ${ }^{1,2}$, WEI-WEI LI ${ }^{1,2}$, CONG-JI WEN ${ }^{1,2}$, YONG-LI DIAO ${ }^{2}$ and TIAN-LAN ZHAO ${ }^{1}$ \\ ${ }^{1}$ Department of Plastic Surgery, The Second Affiliated Hospital of Soochow University, Suzhou, Jiangsu 215004; \\ ${ }^{2}$ Department of Burns and Plastic Surgery, Yancheng City No.1 People's Hospital, Yancheng, Jiangsu 224005, P.R. China
}

Received November 6, 2019; Accepted April 9, 2020

DOI: $10.3892 / \mathrm{mmr} .2020 .11446$

\begin{abstract}
Melanoma is a malignant skin cancer type associated with a high mortality rate, but its treatment is currently not ideal. Both microRNA (miR)-214 and cell adhesion molecule 1 (CADM1) are differentially expressed in melanoma, but their role in this cancer type remains unknown. Therefore, the aim of the present study was to investigate the role of CADM1 and miR-214 in melanoma to identify novel targets for its treatment. The expression levels of CADM1 and miR-214 in cells were detected by reverse transcription-quantitative PCR (RT-qPCR). Moreover, cell viability, migration and invasion were measured by MTT, wound healing and Transwell assays, respectively. In addition, the relative expression levels of epithelial-mesenchymal transition (EMT)-related proteins in cells were detected by RT-qPCR and western blotting. It was found that the expression of CADM1 was inhibited in melanoma cells, while miR-214 expression was increased during melanoma tumorigenesis. Furthermore, miR-214 mimics promoted the viability, migration and invasion of melanoma cells. It was also demonstrated that the downregulation of CADM1 reversed the inhibitory effect of the miR-214 inhibitor in melanoma. Moreover, overexpression of CADM1 inhibited the EMT process in melanoma, while the miR-214 inhibitor suppressed the EMT process. The results also indicated that miR-214 promoted the EMT process by downregulating CADM1, which may represent a novel mechanism for the progression of melanoma.
\end{abstract}

\section{Introduction}

Melanoma is the second most dangerous malignant tumor type and ranks second only to pancreatic cancer (1). Moreover, melanoma is characterized by early metastasis

Correspondence to: Dr Tian-Lan Zhao, Department of Plastic Surgery, The Second Affiliated Hospital of Soochow University, 1055 Sanxiang Road, Suzhou, Jiangsu 215004, P.R. China

E-mail: zhaotianlan5577@163.com

Key words: melanoma, epithelial-mesenchymal transition, cell adhesion molecule 1, microRNA-214 and high mortality rates. In recent years, the incidence of numerous malignant tumors has decreased from 40 to $20 \%$ worldwide, but the incidence of malignant melanoma remains high (2). Epithelial-mesenchymal transition (EMT) has a critical role in the process of cell remodeling during tumorigenesis (3). Furthermore, previous studies have reported that EMT plays a key role in the metastasis of malignant tumors (4-6). However, as the metastasis of melanoma has not been effectively controlled (7), it is important to identify the mechanism via which the EMT process is promoted.

Cell adhesion molecule 1 (CADM1), which is regarded as a suppressor gene in the tumorigenesis of non-small cell lung cancer, has been identified as a diagnostic marker for adult T-cell leukemia and lymphoma (8). In addition, it has been previously reported that CADM1 plays an inhibitory role in the progression, migration and invasion of cancer, such as cervical cancer and liver cancer (9-11), indicating that CADM1 is an inhibitor of tumor cell malignant features. You et al (12) showed that CADM1 was differentially expressed in melanoma, but its function in melanoma remains unknown. Moreover, microRNAs (miRNAs) act as imperfect sequence guides to recruit a ribonucleoprotein complex to the complementary RNA (13). Furthermore, miRNA expression profiles differ between different stages of cancer and healthy tissues, and previous studies have used miRNAs as diagnostic markers, either alone or in combination with other known biomarkers (14-16). Preliminary studies examining miRNA expression also used tissues to determine the functional and diagnostic roles of miRNAs $(13,17,18)$, which have been reported to regulate the development and progression of cancer $(19,20)$. It has also been revealed that miRNAs (miRs) can promote or inhibit the EMT process in cancer types (21-23). For instance, miR-214 is a member of the miRNA family, and it can induce immune suppression and promote the occurrence of cancer (24), thus demonstrating that miR-214 plays a key role in tumorigenesis. In addition, Mirzaei et al (25) revealed that miR-214 is differentially expressed in melanoma. However, the relationship between CADM1 and miR-214 is not fully understood.

Based on these previous findings, the aims of the present study were to investigate the mechanism via which miR-214 regulates the EMT process in melanoma, and to identify the relationship between CADM1 and miR-214. Thus, the 
present results may provide a potential novel target for treating melanoma.

\section{Materials and methods}

Cell lines and cell culture. The PIG-1, WM-266-4, A2058 and A375 cell lines were purchased from the Cell Bank of Chinese Academy of Science, and cultured in 90\% RPMI-1640 (Thermo Fisher Scientific, Inc.) with 10\% FBS (Thermo Fisher Scientific, Inc.) and maintained at $37^{\circ} \mathrm{C}$ in a humidified incubator containing $5 \% \mathrm{CO}_{2}$. The BR-V-108 plasmid vector (Shanghai Biosciences Co., Ltd.) and competent TOP10 E. coli cells (Shanghai Biosciences Co., Ltd.) were used.

Vector construction and cell transfection. RNA interference target sequences were designed using the CADMI gene as a template to construct a target gene RNA interference 5'-CCA CAGGACAATGCTGAACTT-3' lentiviral vector (Shanghai Biosciences Co.,Ltd.). A single-stranded DNA oligo containing the interference sequence was synthesized, and the obtained lyophilized powder was dissolved in an annealing buffer (final concentration, $100 \mathrm{M}$; Beyotime Institute of Biotechnology) and incubated in a water bath at $90^{\circ} \mathrm{C}$ for $15 \mathrm{~min}$. After naturally cooling to room temperature, a double-stranded oligo with overhang ends was formed, which was then directly ligated into the digested lentiviral vector through restriction sites at both ends. A $50 \mu 1$ reaction system was prepared according to the NEB (New England Biolabs, Inc.) instructions, the BR-V-108 vector was linearized by double digestion and the ligation product was introduced into prepared TOP10 E. coli (Tiangen Biotech Co., Ltd.) competent cells. The sequencing results were compared with those of the correct clones and the plasmid was isolated.

miR-214 mimics (upregulation of miR-214, a chemically modified single-stranded RNA that can mimic and enhance the function of endogenous miR-214) and miR-214 inhibitor (downregulation of miR-214, a chemically modified single-stranded RNA that can specifically target miR-214 in melanoma cells), as well as their corresponding negative controls (NCs), were designed by Shanghai GenePharma Co., Ltd. For cell transfection, melanoma cells $\left(1.5 \times 10^{5} /\right.$ well $)$ in a 6 -well plate were transfected with 200 pmol CADM1 short hairpin RNA (shRNA), or 100 pmol miR-214 mimics, inhibitor or their NCs using Lipofectamine ${ }^{\circledR} 2000$ reagent (Thermo Fisher Scientific, Inc.) for $48 \mathrm{~h}$ according to the manufacturer's instructions. Transfected cells were incubated for an additional $24 \mathrm{~h}$ at $4^{\circ} \mathrm{C}$ and then used to test the cell viability. The sequences were as follows: miR-214 mimics, 5'-UGCCUGUCUACACUUGCUGUGC-3'; miR-214 inhibitor, 5'-ACUGCCUGUCUGUGCCUGCUGU-3'; CADM1 siRNA sense, 5'-CACCGCAGATGACTTATCCTCTAC AATTCAAGAGATTGTAGAGGATAAGTCATCTGTTTTT TG-3' and anti-sense, 5'-GATCCAAAAAACAGATGACTT ATCCTCTACAATCTCTTGAATTGTAGAGGATAAGTC ATCTGC-3'.

Reverse transcription-quantitative PCR (RT-qPCR). Total RNA was extracted from melanoma cell lines using TRIzol ${ }^{\circledR}$ reagent (Thermo Fisher Scientific, Inc.) according to the manufacturer's protocol. First-strand cDNA was synthesized using the PrimeScript RT reagent kit (Takara Bio, Inc.) according to the manufacturer's protocol. RT-qPCR was performed in an ABI7500 system (Thermo Fisher Scientific, Inc.) using SYBR-Green (Takara Bio, Inc.) methods. RT-qPCR was performed in triplicate using the following protocol: Initial denaturation for $2 \mathrm{~min}$ at $94^{\circ} \mathrm{C}$, followed by 35 cycles for $30 \mathrm{sec}$ at $94^{\circ} \mathrm{C}$ and $45 \mathrm{sec}$ at $55^{\circ} \mathrm{C}$. The primers for all the genes were obtained from Shanghai GenePharma Co., Ltd. Relative gene expression was quantified using the $2^{-\Delta \Delta \mathrm{Cq}}$ method (26). The primer sequences are listed in Table I.

Mature miRNAs were quantified using miRNA assays. The protocol was as following: cDNA was synthesized from 5-10 $\mu \mathrm{g}$ of total RNA using the microRNA cDNA Synthesis kit (Takara Bio, Inc.). Then, PCR assays using were performed using SYBR premix Ex Taq II kit (Takara Bio, Inc.). RT-qPCR conditions used were as follows: Initial denaturation for $10 \mathrm{~min}$ at $95^{\circ} \mathrm{C}$, followed by 40 cycles of $15 \mathrm{sec}$ at $95^{\circ} \mathrm{C}$ and $1 \mathrm{~min}$ at $60^{\circ} \mathrm{C}$. Relative miRNA and mRNA expression levels were measured and normalized to U6 and GAPDH expression, respectively.

Western blot analysis. To investigate EMT-related protein expression levels, A375 or A2058 cells were collected and lysed using RIPA lysis buffer (Cell Signaling Technology, Inc.) on ice according to the manufacturer's instructions. Total cellular proteins (40 $\mu \mathrm{g} /$ lane) were quantified by bicinchoninic protein assay kit (Beyotime Institute of Biotechnology), and then subjected to $10 \%$ SDS-PAGE for western blotting. Proteins were transferred to PVDF membranes, the blots were incubated at room temperature with 5\% BSA (Thermo Fisher Scientific, Inc.) in TBST $(0.5 \%$ Tween-20) for $60 \mathrm{~min}$ and then incubated overnight at $4^{\circ} \mathrm{C}$ on a rocker with the following primary antibodies: Anti-CADM1 (1:1,000; cat. no. ab3910; Abcam), anti-E-cadherin (1:3,000; cat. no. ab194982; Abcam), anti-vimentin (1:3,000; cat. no. ab92547; Abcam), anti-Slug (1:1,000; cat. no. ab51772; Abcam) and anti-GAPDH (1:3,000; cat. no. ab181602; Bioworld Technology, Inc.). After washing three times with TBST for $5 \mathrm{~min}$, the membranes were incubated with horseradish peroxidase-conjugated goat anti-rabbit IgG polyclonal secondary antibody (1:3,000; ab191866; Beyotime Institute of Biotechnology) at room temperature for $1 \mathrm{~h}$. An ECL ${ }^{+}$western blotting system kit (GE Healthcare Life Science) was used for color development. The density of the bands was measured using ImageJ software (version 6.0; National Institutes of Health). Signals were detected with enhanced chemiluminescence using GAPDH as the internal standard (Kodak).

Dual-luciferase reporter assay. The downstream target of CADM1 was predicted using Starbase 2.0 (Sun Yat-sen University; http://starbase.sysu.edu.cn/). Both wild-type and mutant constructs of CADM1 were cloned into the pmirGLO Dual-Luciferase miRNA target expression vector (Promega Corporation). A 375 or A2058 cells ( $5 \times 10^{3}$ cells per well) were seeded in a 24-well plate and co-transfected with wild-type or mutant CADM1 3'untranslated region and mimic NC or miR-214 mimics using Lipofectamine ${ }^{\circledR} 3000$ (Thermo Fisher Scientific, Inc.). Transfection and harvest efficiencies were controlled using the pmirGLO reporter as an internal control. Cells were collected $48 \mathrm{~h}$ post-transfection and analyzed using the dual-luciferase reporter assay system (Promega 
Table I. Primer sequences used in the present study.

\begin{tabular}{ll}
\hline Gene & \multicolumn{1}{c}{ Sequence } \\
\hline miR-214 & F: 5'-AGCACAGCAGGCACAGACA-3' \\
& R: 5'-CAGTGCGTGTCGTGGAGT-3' \\
U6 & F: 5'-CGCTTCGGCAGCACACATATAC-3' \\
& R: 5'-CAGGGGCCATGCTAATCTT-3' \\
CADM1 & F: 5'-GGTGATGGGCAGAATCTGTT-3' \\
& R: 5'-AGGCCTGAAGTCCCTGAAAT-3' \\
E-cadherin & F: 5'-TCACATCCTACACTGCCCAG-3' \\
& R: 5'-AGTGTCCCTGTTCCAGTAGC-3' \\
Vimentin & F: 5'-GGACCAGCTAACCAACGACA-3' \\
& R: 5'-AAGGTCAAGACGTGCCAGAG-3' \\
Slug & F: 5'-ACGCCTCCAAAAAGCCAAAC-3' \\
& R: 5'-ACAGTGATGGGGCTGTATGC-3' \\
GAPDH & F: 5'-CCACCCATGGCAAATTCCATGGCA-3' \\
& R: 5'-TCTAGACGGCAGGTCAGGTCCAC-3'
\end{tabular}

$\mathrm{F}$, forward; R, reverse; miR, microRNA; CADM1, cell adhesion molecule 1 .

Corporation). The data were quantified via normalizing to Renilla luciferase activity.

MTT assay. An MTT assay was performed to investigate the effect of miR-214 and CADM1 on melanoma cell viability. A 375 and A2058 cells $\left(5 \times 10^{3}\right.$ cells per well) were seeded on 96-well plates and incubated at $37^{\circ} \mathrm{C}$ for 24,48 and $72 \mathrm{~h}$. Then, $20 \mu \mathrm{l}$ MTT solution (Sigma-Aldrich; Merck KGaA) was added to each well and incubated at room temperature for another $4 \mathrm{~h}$. Then, the culture medium with MTT was discarded, $150 \mu \mathrm{l}$ DMSO was added and cells were agitated for $10 \mathrm{~min}$ at room temperature. The absorbance was measured at $490 \mathrm{~nm}$ with a standard microplate reader, and cell viability was analyzed.

Wound healing assay. A 375 and A2058 cells (5x104 cells per well) were plated in a 6 -well plate for culture. When cells were at $90 \%$ confluency, the medium was replaced with RPMI-1640 (Thermo Fisher Scientific, Inc.) for the scratch test, and the slide was pushed up to form a scratch. Then, $0.5 \%$ FBS was added and cells were imaged using a light microscope (magnification, x100). Cells were incubated at $37^{\circ} \mathrm{C}$ in a $5 \% \mathrm{CO}_{2}$ incubator for $48 \mathrm{~h}$ and then assessed using Image $\mathrm{J}$ software (version 6.0; National Institutes of Health). The cell migration rate of each group was calculated based on the images acquired after the scratch.

Transwell assay. For the invasion assay, the upper chamber (polycarbonic membrane; diameter $6.5 \mathrm{~mm}$; pore size, $8 \mu \mathrm{m}$; Corning, Inc.) was pretreated with $100 \mu \mathrm{l}$ Matrigel (BD Biosciences) at $37^{\circ} \mathrm{C}$ for $30 \mathrm{~min}$, and melanoma cells ( $1 \times 10^{5}$ cells) in FBS-free medium (RPMI-1640) were seeded into the upper chamber. The lower chamber contained RPMI-1640 supplemented with 10\% FBS. Then, cells were incubated at $37^{\circ} \mathrm{C}$ for $24 \mathrm{~h}$. The cells that attached to the underside of the membrane were fixed using $5 \%$ glutaraldehyde at $4{ }^{\circ} \mathrm{C}$ for $30 \mathrm{~min}$ and stained with a $0.5 \%$ crystal violet solution at room temperature for $30 \mathrm{~min}$. Subsequently, images were captured and the number of invading cells was counted under a light microscope (magnification, x100).

Statistical analysis. All assays were performed in $\geq 3$ independent experiments, and data are presented as the mean \pm SD. Comparison between two groups was analyzed using unpaired Student's t-test. Comparisons among multiple groups were performed with one-way ANOVA followed by Tukey's test, using GraphPad Prism 7 (version 7; GraphPad Software, Inc.). $\mathrm{P}<0.05$ was considered to indicate a statistically significant difference.

\section{Results}

CADM1 has an inhibitory effect in melanoma. To investigate the role of CADM1 in melanoma cells, the expression of CADM1 was detected using RT-qPCR. It was found that the expression of CADM1 was significantly decreased in melanoma cells (WM-266-4, A2058 and A375) compared with normal melanocytes cells (PIG1; Fig. 1A). Western blotting was then used to assess this result, and it was identified that the protein expression of CADM1 was significantly inhibited in the melanoma cells compared with PIG1 cells (Fig. 1B). Therefore, these results indicated that CADM1 had an inhibitory effect in melanoma in vitro.

Next, the efficiency of cell transfection was detected by RT-qPCR. The results suggested that the expression of CADM1 in the melanoma cells was significantly upregulated by overexpression of CADM1, but decreased in the presence of short hairpin (sh)-CADM1 (Fig. 1C). Then, to examine the effect of CADM1 on the EMT process of melanoma, RT-qPCR and western blotting were performed. The results demonstrated that the relative expression levels of vimentin and Slug were significantly inhibited by overexpression of CADM1, but increased by CADM1 knockdown. However, the expression of E-cadherin in melanoma cells was significantly increased after CADM1 overexpression, while knockdown of CADM1 significantly decreased the relative expression of E-cadherin (Fig. 1D and E). Collectively, these results demonstrated that overexpression of CADM1 significantly downregulated the EMT process in melanoma cells, while reducing the expression of CADM1 had the opposite effect.

miR-214 significantly upregulates the progression of melanoma in vitro. RT-qPCR was used to investigate the role of miR-214 in melanoma, and it was identified that the expression of miR-214 was significantly increased in melanoma cells compared with normal cells (Fig. 2A). In addition, miR-214 expression was increased to the highest level in A375 cells. Next, RT-qPCR was used to assess the cell transfection and the results indicated that miR-214 was significantly upregulated in melanoma cells in the presence of miR-214 mimics. However, the expression of miR-214 in melanoma cells was significantly decreased by miR-214 inhibitor (Fig. 2B).

To further examine the effect of miR-214 on the tumorigenesis of melanoma, an MTT assay was used to measure the viability of melanoma cells. The results suggested that 
A

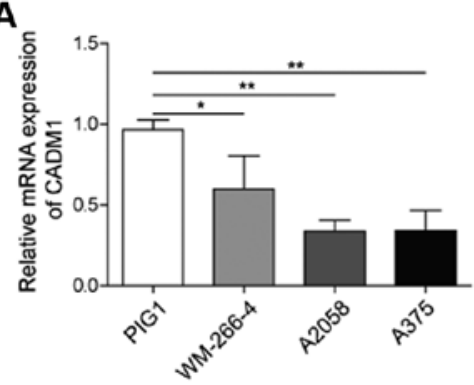

C

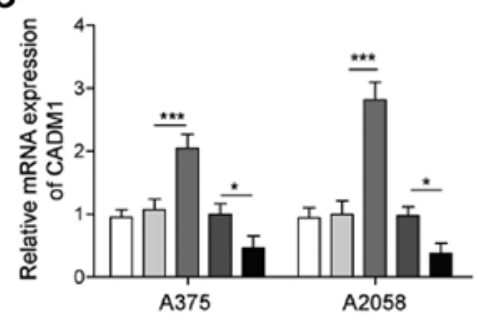

B

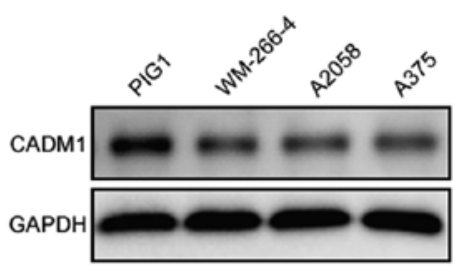

D
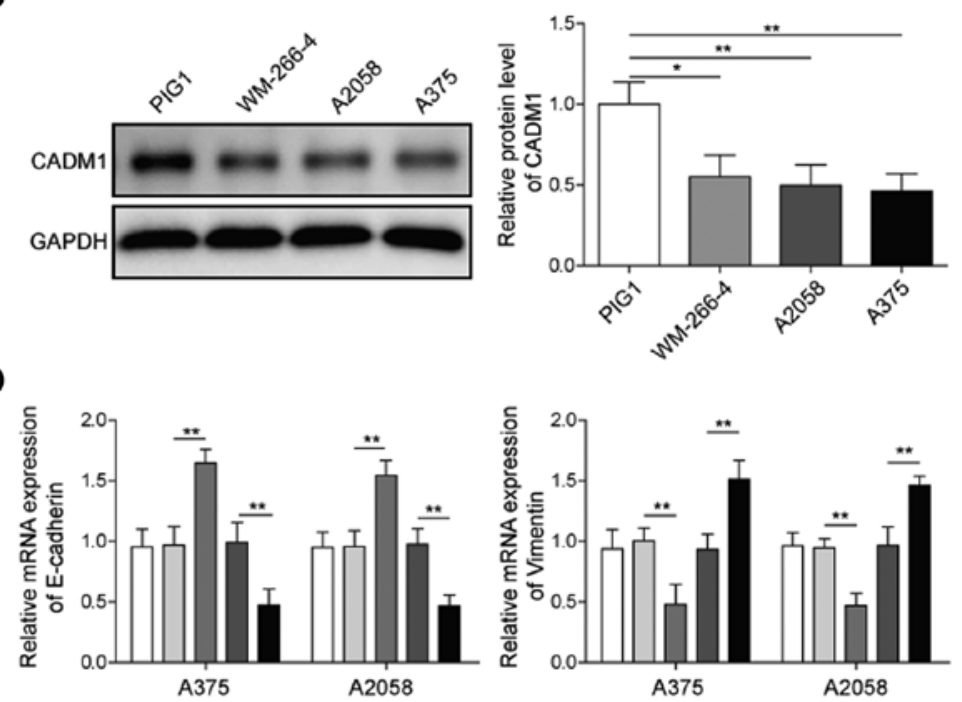

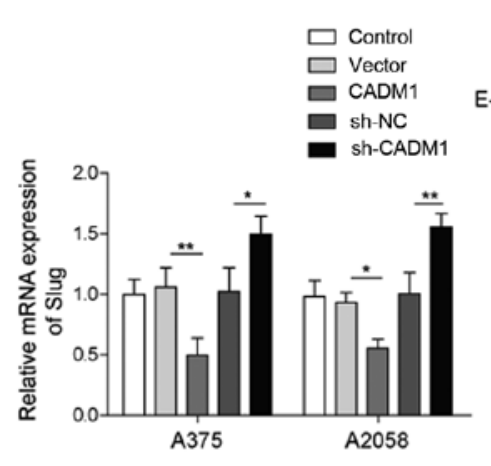

E
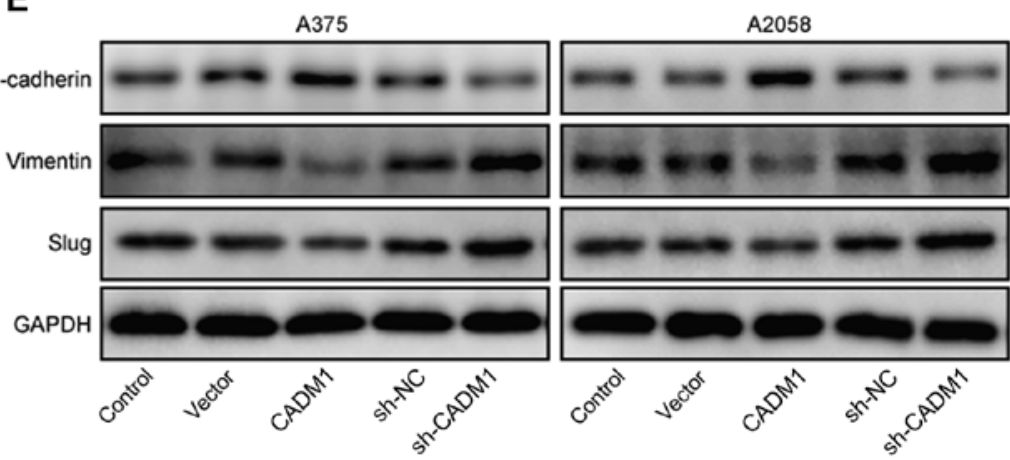

$\square$ Control

CADM

$\square$ sh-NC
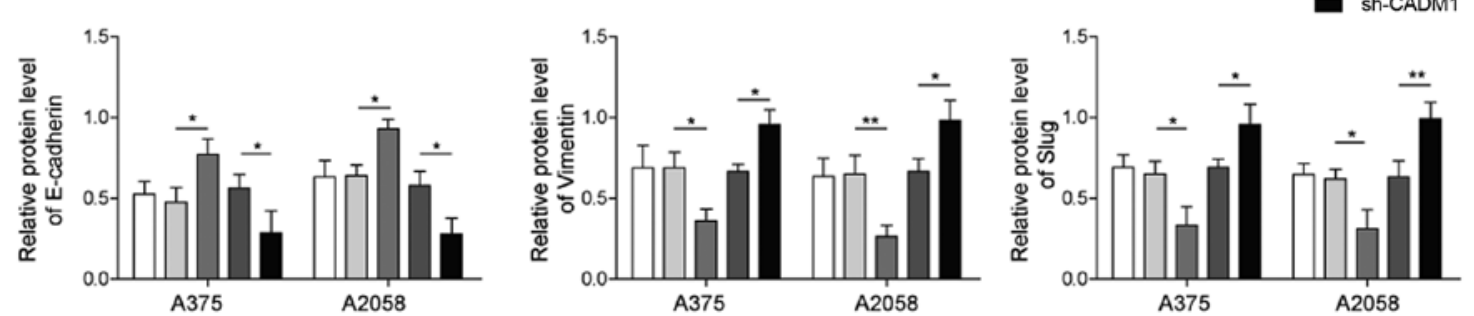

Figure 1. CADM1 downregulates the EMT process in melanoma. Relative expression of CADM1 in PIG1, WM-266-4, A2058 and A375 cells was detected by (A) RT-qPCR and (B) western blotting. (C) A375 or A2058 cells were transfected with overexpression of CADM1, NC or sh-CADM1 for 24 h. Then, cell transfection was assessed by RT-qPCR. Relative expression levels of EMT-related proteins in melanoma cells were detected by (D) RT-qPCR and (E) western blotting. There were $\geq 3$ independent experiments performed for each group. ${ }^{*} \mathrm{P}<0.05,{ }^{* *} \mathrm{P}<0.01,{ }^{* * *} \mathrm{P}<0.001$. sh, short hairpin $\mathrm{RNA}$; NC, negative control; miR, microRNA; CADM1, cell adhesion molecule 1; RT-qPCR, reverse transcription-quantitative PCR; EMT, epithelial-mesenchymal transition.

the viability of A375 cells was significantly increased in the presence of miR-214 mimic, which was reversed by miR-214 inhibitor (Fig. 2C). Moreover, to investigate the role of miR-214 in the progression of melanoma in vitro, wound healing and Transwell assays were performed to detect the migration and invasion of melanoma cells, respectively. It was found that the miR-214 mimic significantly increased the migration of melanoma cells, while the miR-214 inhibitor significantly inhibited the migration of A375 and A2058 cells (Fig. 2D). Furthermore, during the invasion assay, A375 cells treated with miR-214 mimic exhibited a significant invasive ability. However, the miR-214 inhibitor significantly inhibited the invasive ability of melanoma cells (Fig. 2E). Therefore, the results demonstrated that miR-214 overexpression significantly promoted the tumorigenesis and progression of melanoma in vitro, while downregulation of miR-214 exhibited an inhibitory effect.

miR-214 overexpression significantly downregulates the expression of CADM1. To investigate the relationship between miR-214 and CADM1, the binding site of CADM1 and miR-214 was analyzed by bioinformatics prediction software, and a dual-luciferase reporter assay was performed to verify the specific binding of miR-214 and CADM1. Luciferase reporter analysis results identified that miR-214 mimic significantly downregulated the activity of the reporter containing wild-type 
A

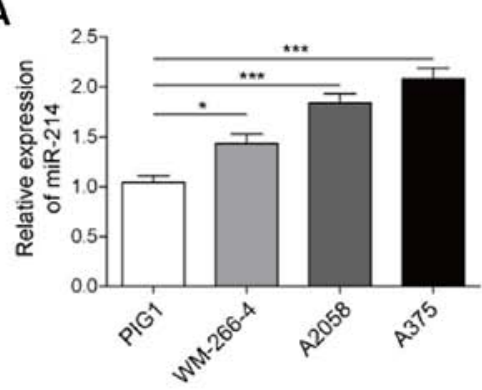

C

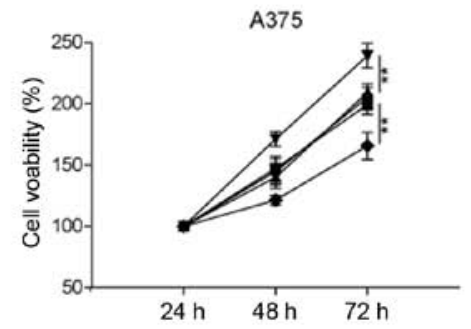

B
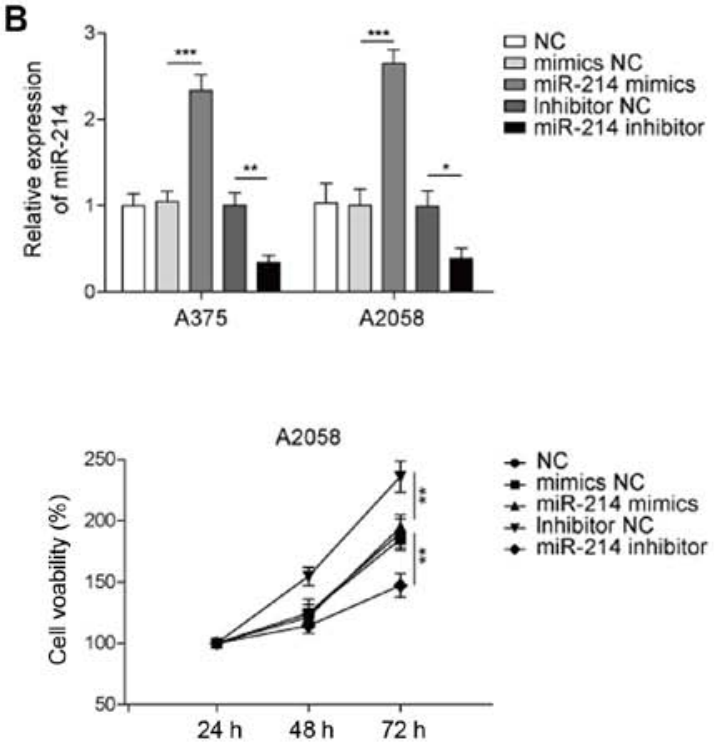

D
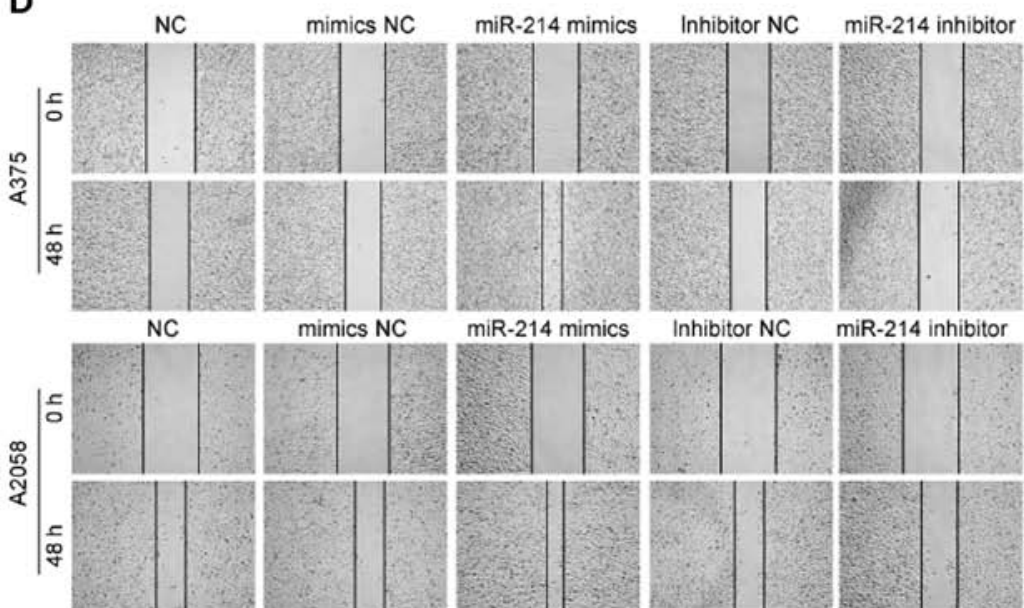

Inhibitor NC miR-214 inhibitor

E

NC

mimics NC
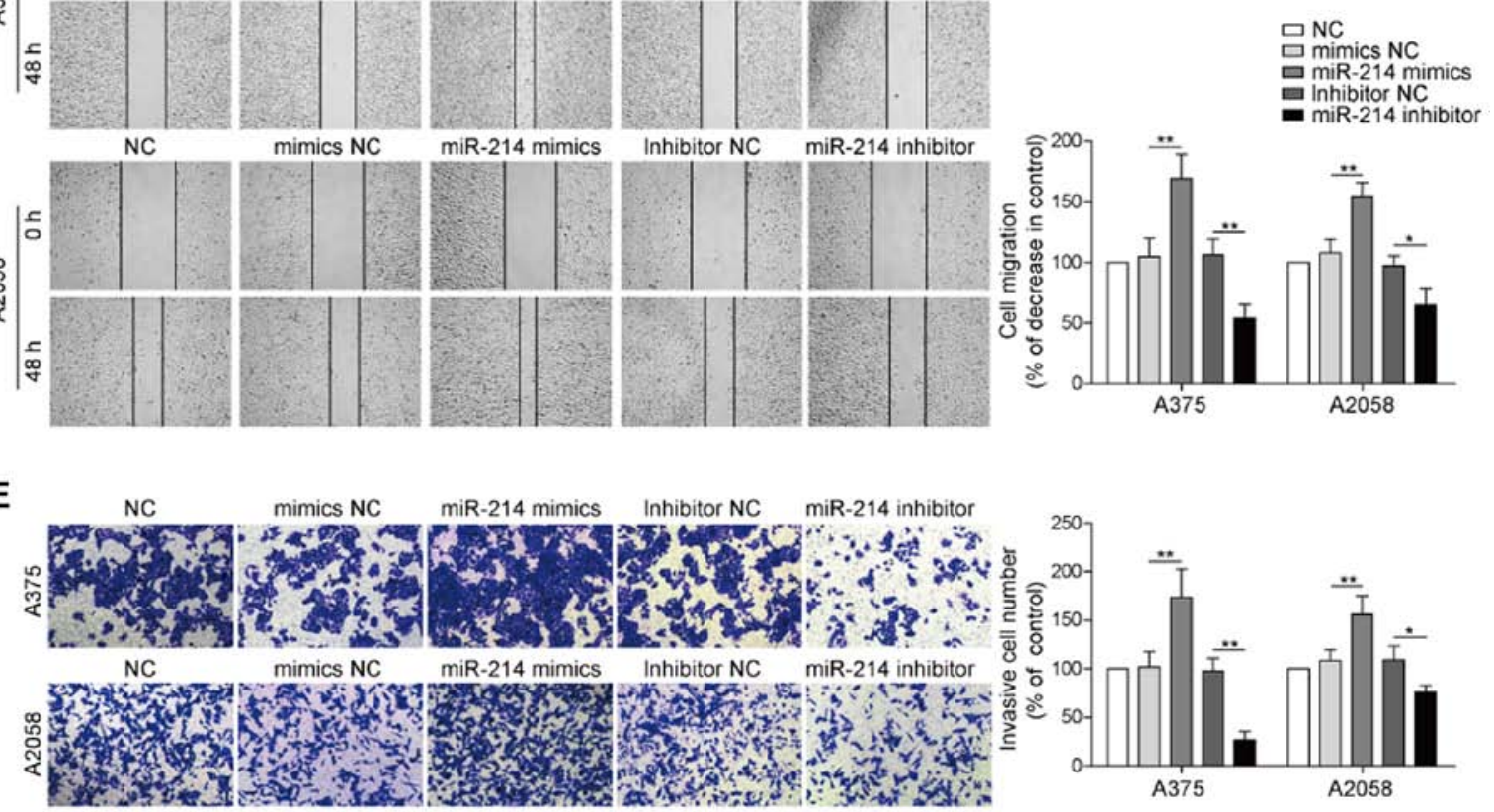

Figure 2. miR-214 upregulates the tumorigenesis and progression of melanoma. (A) Relative expression of miR-214 in PIG1, WM-266-4, A2058 and A375 cells was detected by RT-qPCR. (B) A375 or A2058 cells were transfected with miR-214 mimics, NC or miR-214 inhibitor for 24 h. Then, cell transfection was assessed by RT-qPCR. (C) Cell viability in the NC, mimic NC, inhibitor NC, miR-214 mimic and miR-214 inhibitor groups was measured after 24,48 and $72 \mathrm{~h}$ using MTT assay. (D) Cell migration in the NC, mimic NC, inhibitor NC, miR-214 mimic and miR-214 inhibitor groups was tested by wound healing assay (magnification, $\mathrm{x} 100$ ). (E) Cell invasion in the NC, mimic NC, inhibitor NC, miR-214 mimic and miR-214 inhibitor groups was tested by Transwell assay (magnification, x100). NC, negative control. There were $\geq 3$ independent experiments performed for each group. ${ }^{*} \mathrm{P}<0.05$, ${ }^{* *} \mathrm{P}<0.01,{ }^{* * *} \mathrm{P}<0.001$. NC, negative control; miR, microRNA; RT-qPCR, reverse transcription-quantitative PCR.

CADM1; however, mutating the binding site of miR-214 in CADM1 abolished this suppression (Fig. 3A and B). Thus, the results suggested that CADM1 may be a direct target regulated by miR-214.

In addition, RT-qPCR was used to determine the expression of CADM1 in melanoma cells transfected with miR-214 mimic or inhibitor. It was found that the expression of CADM1 was significantly enhanced by the miR-214 inhibitor, but was reversed by miR-214 mimic (Fig. 3C). Moreover, the results indicated that the protein expression of CADM1 was significantly inhibited by the presence of miR-214 mimic, which was reversed by miR-214 inhibitor (Fig. 3D). Therefore, it was suggested that the overexpression of miR-214 downregulated the expression of CADM1 in melanoma cells, while downregulation of miR-214 increased the expression of CADM1.

miR-214 promotes EMT in melanoma cells by downregulating $C A D M 1$ expression. To further investigate the mechanism 
A

CADM1 3'UTR WT

miR-214-3p

CADM1 3'UTR MUT
5'...CCAUUUUGGAAUUUGCCUGCUGG...3'

3'...UGACGGACAGACACGGACGACA...5'

5'...CCAUUUUGGAAUUU-CGGACGACG...3'

B
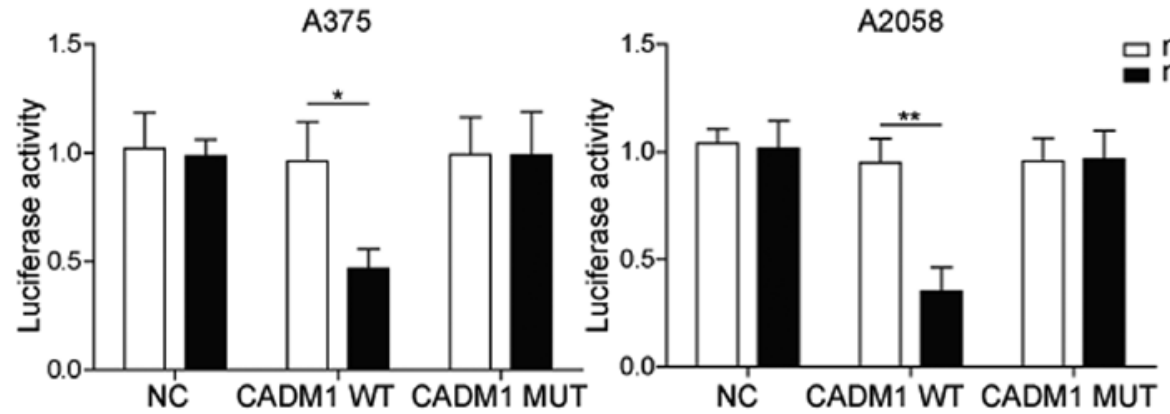

C

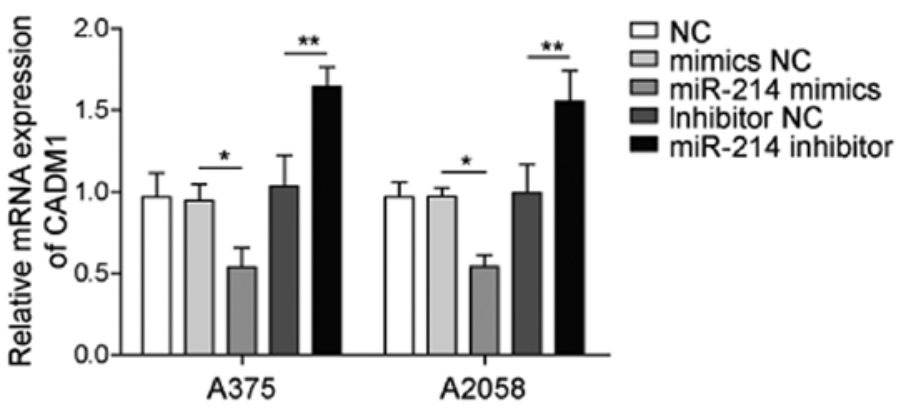

D

A375
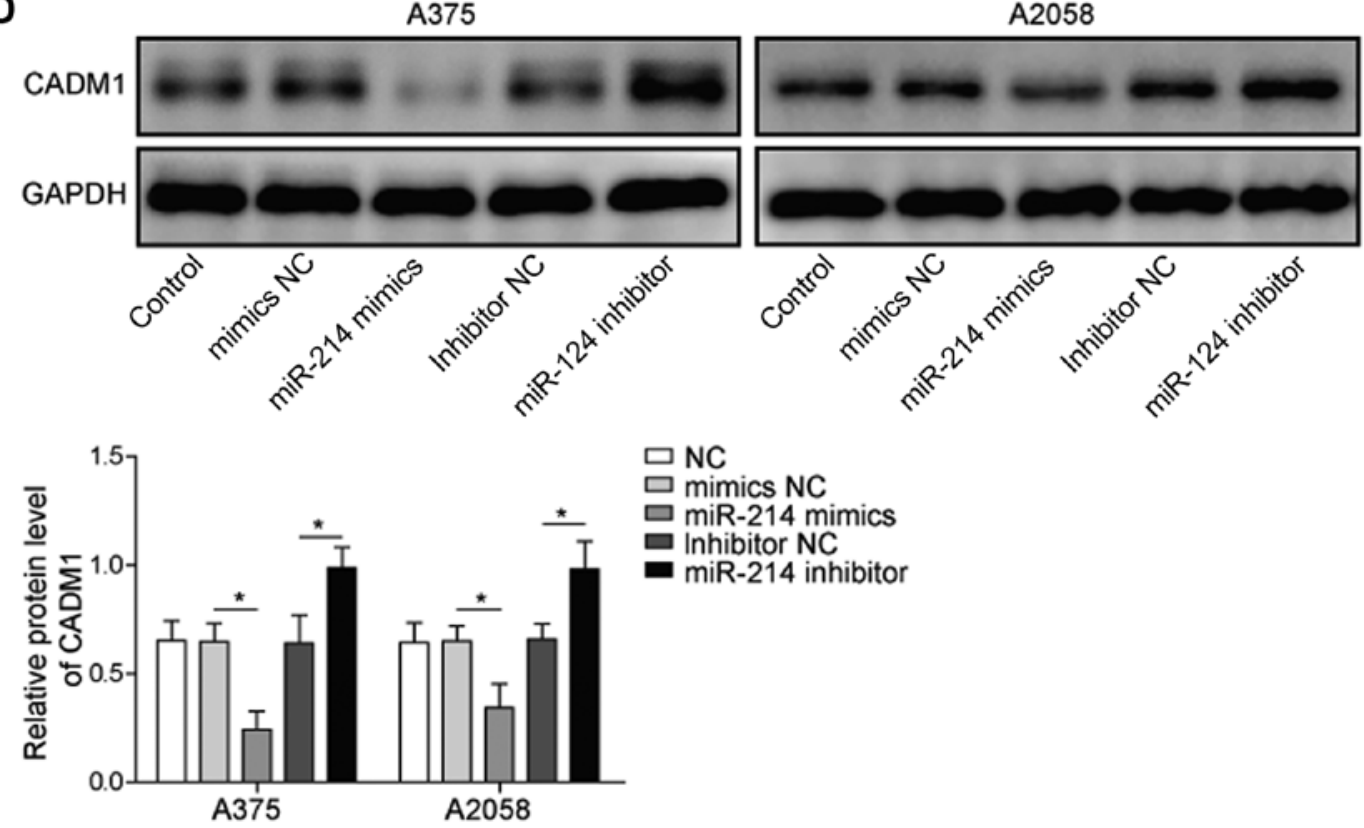

Figure 3. miR-214 regulates CADM1 expression. (A) Binding site of miR-214 to CADM1 was analyzed by bioinformatics prediction software. The position and sequences of the predicted binding sites are shown. (B) A luciferase reporter assay was performed to assess the predicted binding sites with the sequence in miR-214 mimic-transfected melanoma cells. Relative expression of CADM1 in the NC, miR-214 mimic and miR-214 inhibitor groups was detected by (C) reverse transcription-quantitative PCR and (D) western blotting. There were $\geq 3$ independent experiments performed for each group. ${ }^{*} \mathrm{P}<0.05,{ }^{* *} \mathrm{P}<0.01$. NC, negative control; miR, microRNA; WT, wild-type; MUT, mutant; CADM1, cell adhesion molecule 1.

via which miR-214 regulated the EMT process of melanoma in vitro, the expression levels of CADM1 and EMT-related proteins were detected by RT-qPCR and western blotting. It was demonstrated that the expression of CADM1 was significantly upregulated in the presence of miR-214 inhibitor (Fig. 4A and B). Moreover, the miR-214 inhibitor significantly decreased the relative expression levels of vimentin and Slug, but increased the relative expression of E-cadherin in melanoma cells, which was significantly reversed by the knockdown of CADM1.

Furthermore, to investigate the effect of CADM1 on melanoma cells transfected with miR-214 inhibitor, wound healing 
A

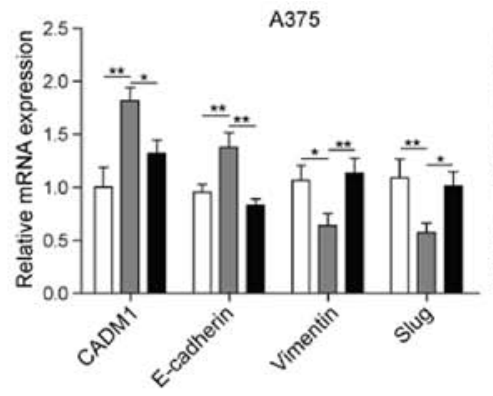

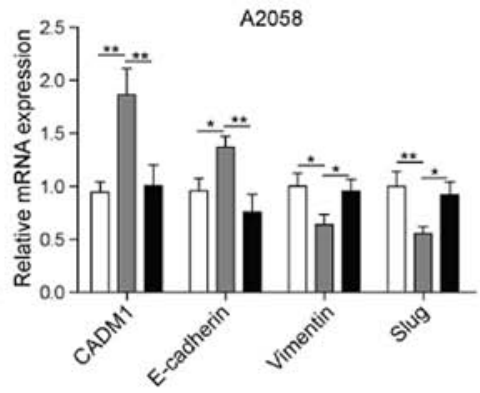

B
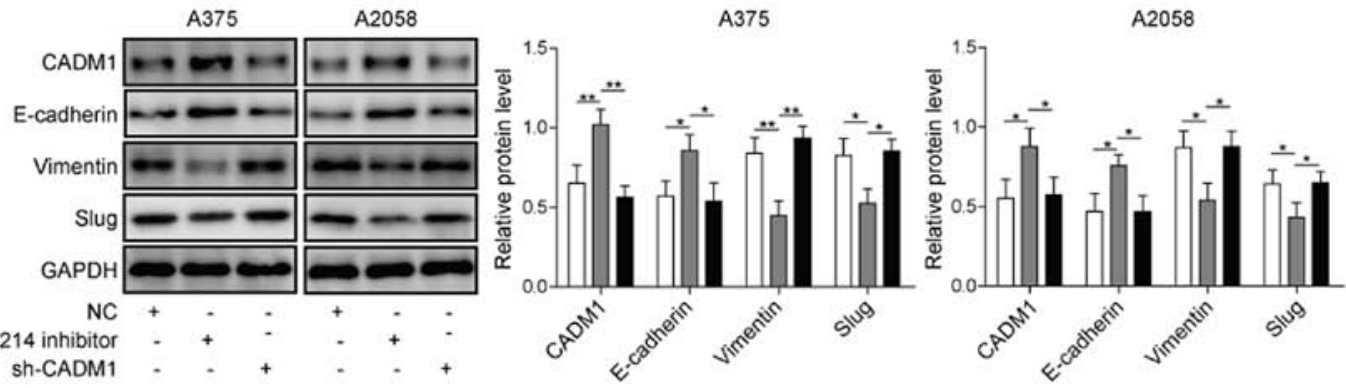

C

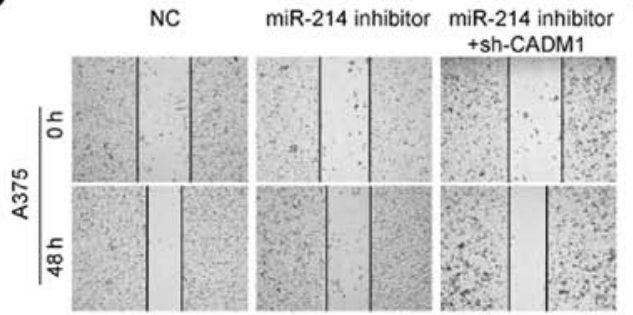

D
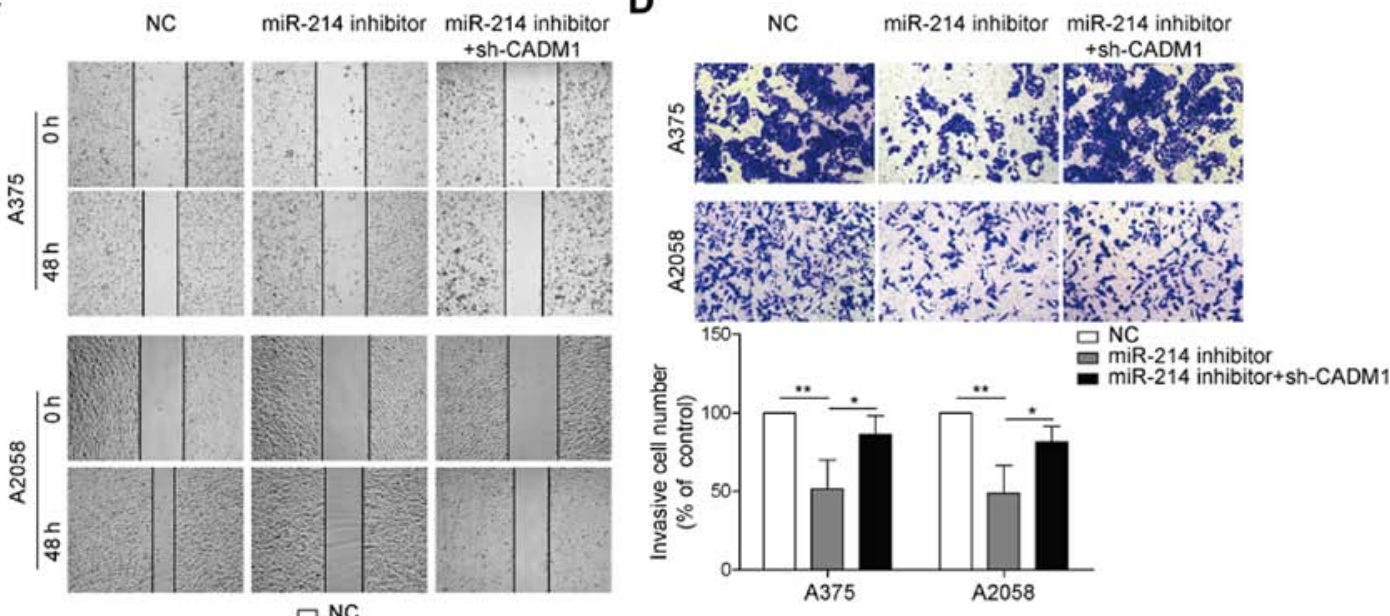

믐 ${ }_{\text {miR-2 }}^{N}$-214 inhibitor

E
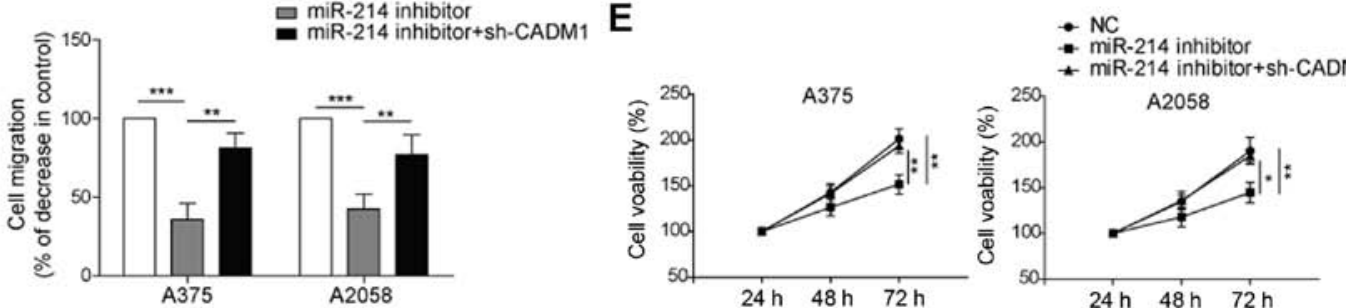

Figure 4. miR-214 downregulates CADM1 and upregulates the EMT process in melanoma cells. (A) Relative mRNA expression levels of E-cadherin, Slug and vimentin in the NC, miR-214 inhibitor and sh-CADM1 + miR-214 inhibitor groups were detected by reverse transcription-quantitative PCR. (B) Relative protein expression levels of EMT-related proteins in the NC, miR-214 inhibitor and sh-CADM1 + miR-214 inhibitor groups were detected by western blotting. (C) Cell migration in the NC, miR-214 inhibitor and sh-CADM1 + miR-214 inhibitor groups was assessed by wound healing assay (magnification, x100). (D) Cell invasion in the NC, miR-214 inhibitor and sh-CADM1 + miR-214 inhibitor groups was measured by Transwell assay (magnification, $\mathrm{x} 100)$. (E) Cell viability in the NC, miR-214 inhibitor and sh-CADM1 + miR-214 inhibitor groups was examined after 24, 48 and $72 \mathrm{~h}$ by MTT assay. There were $\geq 3$ independent experiments performed for each group. ${ }^{*} \mathrm{P}<0.05,{ }^{* * *} \mathrm{P}<0.01,{ }^{* * * *} \mathrm{P}<0.001$. NC, negative control; miR, microRNA; WT, wild-type; MUT, mutant; CADM1, cell adhesion molecule 1; sh, short hairpin RNA; EMT, epithelial-mesenchymal transition.

and Transwell assays were performed. The results demonstrated that the migration of A375 cells was significantly inhibited by miR-214 inhibitor, which was partially rescued by downregulation of CADM1 (Fig. 4C). In the Transwell assay, the invasion of A375 and A2058 cells was significantly inhibited in the presence of miR-214 inhibitor. However, CADM1 downregulation partially reversed the inhibitory effect of miR-214 inhibitor on cell invasion in melanoma
(Fig. 4D). Moreover, to test the effect of CADM1 on the viability of A375 or A2058 cells treated with miR-214 inhibitor, an MTT assay was conducted. It was found that cell viability was decreased by the miR-214 inhibitor, which was partially reversed by knockdown of CADM1 (Fig. 4E). Collectively, the results indicated that miR-214 affected the EMT process of melanoma by modulating the expression of CADM1. 


\section{Discussion}

Previous studies have focused on the metastasis of malignant tumors (27-29); however, the understanding of the mechanism of melanoma development remains limited (30). It has been reported that the immune system plays a key role in the treatment of metastasis in advanced cancer (31-36). Moreover, E-cadherin, vimentin and Slug are key regulators during the EMT process of malignant tumors $(37,38)$. Previous studies have also shown that EMT-related proteins are closely associated with tumor metastasis $(39,40)$, which is in line with the present results, thus indicating that EMT is closely related to tumor metastasis. Furthermore, it is possible that metastatic cells after EMT are vulnerable to host immunosurveillance (41). Consistent with these findings, the present results suggested that metastasis in melanoma may be closely associated with the EMT process, and thus EMT may play a critical role in the metastasis of melanoma.

It has been reported that CADM1 plays an inhibitory role in tumorigenesis and progression of cancer. For instance, overexpression of CADM1 inhibits cell-cycle progression and invasion of hepatocellular carcinoma (11). Moreover, the present results indicated that CADM1 acted as a tumor suppressor in melanoma, which was similar to the results of a previous study (9). Furthermore, overexpression of CADM1 significantly inhibited the EMT process of melanoma. Therefore, it was speculated that CADM1 may inhibit the tumorigenesis and progression of melanoma by downregulating the EMT process.

miR-214 functions as a pro-metastatic factor in malignant tumors (42), and plays a critical role in the migration and invasion of cancer cells (43-45). The present study identified a potential link between miR-214 and CADM1 in the coordination of metastasis in melanoma. Furthermore, the present results suggested that CADM1 expression decreased when miR-214 was upregulated in melanoma cell lines. It was also found that miR-214 significantly promoted the EMT process of melanoma, while CADM1 inhibited the EMT process. These results were similar to those of previous studies (46-48), thus indicating that miR-214 increased the invasion and migration of melanoma by downregulating CADM1 to promote the EMT process. However, it has also been revealed that miR-214 promotes cancer progression by activating the NF-kB signaling pathway (49). Therefore, future studies should focus on the association between melanoma and the NF- $\mathrm{kB}$ signaling pathway. In addition, the present study used $0.5 \%$ FBS to test the cell migration in wound healing assay, which may be a limitation. Although $0.5 \%$ FBS may enhance the proliferative ability of cells to a certain extent, the effect of proliferation on scratches may be negligible due to the low concentration, however, in order to further eliminate this interference, follow-up studies will use other methods to detect cell migration ability.

In conclusion, the present results suggest that miR-214 promoted the EMT process of melanoma by downregulating CADM1, which may serve as a potential novel target for the treatment of melanoma.

\section{Acknowledgements}

Not applicable.

\section{Funding}

No funding was received.

\section{Availability of data and materials}

All data generated or analyzed during this study are included in this published article.

\section{Authors' contributions}

SJW, WWL and TLZ conceived and supervised the study. SJW and TLZ designed the study. CJW and YLD performed the experiments and analyzed the data. All authors reviewed the results and approved the final version of the manuscript.

\section{Ethics approval and consent to participate}

Not applicable.

\section{Patient consent for publication}

Not applicable.

\section{Competing interests}

The authors declare that they have no competing interests.

\section{References}

1. Pavri SN, Clune J, Ariyan S and Narayan D: Malignant melanoma: Beyond the basics. Plast Reconstr Surg 138: 330e-340e, 2016.

2. Rastrelli M, Tropea S, Rossi CR and Alaibac M: Melanoma: Epidemiology, risk factors, pathogenesis, diagnosis and classification. In Vivo 28: 1005-1011, 2014.

3. Xue YB, Ding MQ, Xue L and Luo JH: CircAGFG1 sponges miR-203 to promote EMT and metastasis of non-small-cell lung cancer by upregulating ZNF281 expression. Thorac Cancer 10: 1692-1701, 2019

4. Zhao C, Ling X, Li X, Hou X and Zhao D: MicroRNA-138-5p inhibits cell migration, invasion and EMT in breast cancer by directly targeting RHBDD1. Breast Cancer 26: 817-825, 2019.

5. Ribatti D, Tamma R and Annese T: Epithelial-mesenchymal transition in cancer: A historical overview. Transl Oncol 13: 100773, 2020.

6. Wang F, Xia H and Yao S: Regulatory T cells are a double-edged sword in pulmonary fibrosis. Int Immunopharmacol 84: 106443, 2020.

7. Mansoori B, Mohammadi A, Naghizadeh S, Gjerstorff M, Shanehbandi D, Shirjang S, Najafi S, Holmskov U, Khaze V, Duijf PHG and Baradaran B: miR-330 suppresses EMT and induces apoptosis by downregulating HMGA2 in human colorectal cancer. J Cell Physiol 235: 920-931, 2019.

8. Yuki A, Shinkuma S, Hayashi R, Fujikawa H, Kato T, Homma E, Hamade Y, Onodera O, Matsuoka M, Shimizu H, et al: CADM1 is a diagnostic marker in early-stage mycosis fungoides: Multicenter study of 58 cases. J Am Acad Dermatol 79: 1039-1046, 2018.

9. Hartsough EJ, Weiss MB, Heilman SA, Purwin TJ, Kugel CH III, Rosenbaum SR, Erkes DA, Tiago M, HooKim K, Chervoneva I and Aplin AE: CADM1 is a TWIST1-regulated suppressor of invasion and survival. Cell Death Dis 10: 281, 2019.

10. Rong G, Zhang M, Xia W, Li D, Miao J and Wang H: Plasma CADM1 promoter hypermethylation and D-dimer as novel metastasis predictors of cervical cancer. J Obstet Gynaecol Res 45: 1251-1259, 2019.

11. Wang F, Qi X, Li Z, Jin S, Xie Y and Zhong H: lncRNA CADM1-AS1 inhibits cell-cycle progression and invasion via PTEN/AKT/GSK-3 $\beta$ axis in hepatocellular carcinoma. Cancer Manag Res 11: 3813-3828, 2019. 
12. You Y, Zhang J, Li Y, Li Y, Shi G, Ma L and Wei $\mathrm{H}$ : CADM1/TSLC1 inhibits melanoma cell line A375 invasion through the suppression of matrix metalloproteinases. Mol Med Rep 10: 2621-2626, 2014.

13. Mohr AM and Mott JL: Overview of microRNA biology. Semin Liver Dis 35: 3-11, 2015.

14. Guo J, Jin H, Xi Y, Guo J, Jin Y and Jiang D: The miR-582/CD1B axis is involved in regulation of dendritic cells and is associated with clinical outcomes in advanced lung adenocarcinoma. Biomed Res Int 2020: 4360930, 2020.

15. Nayak B, Khan N, Garg H, Rustagi Y, Singh P, Seth A, Dinda AK and Kaushal S: Role of miRNA-182 and miRNA-187 as potential biomarkers in prostate cancer and its correlation with the staging of prostate cancer. Int Braz J Urol 46: 614-623, 2020.

16. Sun $X, X u W$, Zang $C$ and Li N: miRNA-520c-3p accelerates progression of nasopharyngeal carcinoma via targeting RAB22A. Oncol Lett 19: 771-776, 2020.

17. Tiwari A, Mukherjee B and Dixit M: MicroRNA key to angiogenesis regulation: MiRNA biology and therapy. Curr Cancer Drug Targets 18: 266-277, 2018

18. Lu TX and Rothenberg ME: MicroRNA. J Allergy Clin Immunol 141: 1202-1207, 2018.

19. Chen S, Yang C, Sun C, Sun Y, Yang Z, Cheng S and Zhuge B: miR-21-5p suppressed the sensitivity of hepatocellular carcinoma cells to cisplatin by targeting FASLG. DNA Cell Biol 38 865-873, 2019.

20. Guo HL, Chen LD, Li W, Liang JY, Zhang JC, Li X, Xie XY Lu MD, Kuang M and Wang W: Ultrasomics for early evaluation of the tumor response to microRNA-122 in a nude mouse hepatocellular carcinoma model. J Ultrasound Med 39: 61-71, 2019.

21. Lin T, Hou PF, Meng S, Chen F, Jiang T, Li ML, Shi ML, Liu JJ, Zheng JN and Bai J: Emerging roles of p53 related lncRNAs in cancer progression: A systematic review. Int J Biol Sci 15 : $1287-1298,2019$

22. Chen Z, Zhuang W, Wang Z, Xiao W, Don W, Li X and Chen X: MicroRNA-450b-3p inhibits cell growth by targeting phosphoglycerate kinase 1 in hepatocellular carcinoma. J Cell Biochem 120: 18805-18815, 2019.

23. Chen J, Lin Y, Jia Y, Xu T, Wu F and Jin Y: LncRNA HAND2-AS1 exerts anti-oncogenic effects on ovarian cancer via restoration of BCL2L11 as a sponge of microRNA-340-5p. J Cell Physiol 234: 23421-23436, 2019.

24. Yin Y, Cai X, Chen X, Liang H, Zhang Y, Li J, Wang Z, Chen X, Zhang W, Yokoyama S, et al: Tumor-secreted miR-214 induces regulatory T cells: A major link between immune evasion and tumor growth. Cell Res 24: 1164-1180, 2014.

25. Mirzaei H, Gholamin S, Shahidsales S, Sahebkar A, Jaafari MR, Mirzaei HR, Hassanian SM and Avan A: MicroRNAs as potential diagnostic and prognostic biomarkers in melanoma. Eur J Cancer 53: 25-32, 2016

26. Livak KJ and Schmittgen TD: Analysis of relative gene expression data using real-time quantitative PCR and the 2(-Delta Delta C(T)) method. Methods 25: 402-408, 2001.

27. Uketa S, Shimizu Y, Ogawa K, Utsunomiya N, Katsushima H, Ishihara M, Hashimoto K and Kanamaru S: A case of isolated peritoneal metastasis from prostate cancer. Hinyokika Kiyo 65: 175-179, 2019 (In Japanese).

28. Shi J, Lu P, Shen W, He R, Yang MW, Fang Y, Sun YW, Niu N and Xue J: CD90 highly expressed population harbors a stemness signature and creates an immunosuppressive niche in pancreatic cancer. Cancer Lett 453: 158-169, 2019.

29. Nakano K, Masui T, Yogo A, Uchida Y, Sato A, Kasai Y, Nagai K, Anazawa T, Kawaguchi Y and Uemoto S: Chloroquine induces apoptosis in PanNEN via endoplasmic reticulum stress. Endocr Relat Cancer 2020.

30. Shen C, Hua H, Gu L, Cao S, Cai H, Yao X and Chen X: miR-124 functions as a melanoma tumor suppressor by targeting RACK1. Onco Targets Ther 12: 9975-9986, 2019.

31. Chockley PJ, Chen J, Chen G, Beer DG, Standiford TJ and Keshamouni VG: Epithelial-mesenchymal transition leads to NK cell-mediated metastasis-specific immunosurveillance in lung cancer. J Clin Invest 128: 1384-1396, 2018.

32. Slaney CY, Rautela J and Parker BS: The emerging role of immunosurveillance in dictating metastatic spread in breast cancer. Cancer Res 73: 5852-5857, 2013.

33. Hong H, Gu Y, Zhang H, Simon AK, Chen X, Wu C, Xu XN and Jiang S: Depletion of CD4+CD25+ regulatory T cells enhances natural killer $\mathrm{T}$ cell-mediated anti-tumour immunity in a murine mammary breast cancer model. Clin Exp Immunol 159: 93-99, 2010.
34. Olkhanud PB, Baatar D, Bodogai M, Hakim F, Gress R, Anderson RL, Deng J, Xu M, Briest S and Biragyn A: Breast cancer lung metastasis requires expression of chemokine receptor CCR4 and regulatory T cells. Cancer Res 69: 5996-6004, 2009.

35. Paolino M, Choidas A, Wallner S, Pranjic B, Uribesalgo I, Loeser S, Jamieson AM, Langdon WY, Ikeda F, Fededa JP, et al: The E3 ligase Cbl-b and TAM receptors regulate cancer metastasis via natural killer cells. Nature 507: 508-512, 2014

36. Yang L, Huang J, Ren X, Gorska AE, Chytil A, Aakre M, Carbone DP, Matrisian LM, Richmond A, Lin PC and Moses HL: Abrogation of TGF beta signaling in mammary carcinomas recruits $\mathrm{Gr}-1+\mathrm{CD} 11 \mathrm{~b}+$ myeloid cells that promote metastasis. Cancer Cell 13: 23-35, 2008.

37. Wei CY, Zhu MX, Yang YW, Zhang PF, Yang X, Peng R, Gao C, Lu JC, Wang L, Deng XY, et al: Downregulation of RNF128 activates Wnt/ $\beta$-catenin signaling to induce cellular EMT and stemness via CD44 and CTTN ubiquitination in melanoma. J Hematol Oncol 12: 21, 2019.

38. Pearlman RL, Montes de Oca MK, Pal HC and Afaq F: Potential therapeutic targets of epithelial-mesenchymal transition in melanoma. Cancer Lett 391: 125-140, 2017.

39. Wang C, Li K, Men Y, Ding C, Du J, Liang T, Ji Z, Chen L, Wang $\mathrm{T}$ and Kang Q: Protein 4.1B suppresses tumor metastasis by regulating epithelial-mesenchymal transition progression in melanoma cells. Int J Med Sci 16: 529-536, 2019.

40. Wels C, Joshi S, Koefinger P, Bergler H and Schaider H: Transcriptional activation of ZEB1 by Slug leads to cooperative regulation of the epithelial-mesenchymal transition-like phenotype in melanoma. J Invest Dermatol 131: 1877-1885, 2011.

41. Bie Y, Ge W, Yang Z, Cheng X, Zhao Z, Li S, Wang W, Wang Y, Zhao X, Yin Z and Li Y: The crucial role of CXCL8 and its receptors in colorectal liver metastasis. Dis Markers 2019: $8023460,2019$.

42. Penna E, Orso F, Cimino D, Vercellino I, Grassi E, Quaglino E, Turco E and Taverna D: miR-214 coordinates melanoma progression by upregulating ALCAM through TFAP2 and miR-148b downmodulation. Cancer Res 73: 4098-4111, 2013.

43. Maity S, Das F, Ghosh-Choudhury N, Kasinath BS and Ghosh Choudhury G: High glucose increases miR-214 to power a feedback loop involving PTEN and the Akt/mTORC1 signaling axis. FEBS Lett 593: 2261-2272, 2019.

44. Gong L, Xu H, Zhang X, Zhang T, Shi J and Chang H: Oridonin relieves hypoxia-evoked apoptosis and autophagy via modulating microRNA-214 in H9c2 cells. Artif Cells Nanomed Biotechnol 47: 2585-2592, 2019.

45. Eguchi S, Takefuji M, Sakaguchi T, Ishihama S, Mori Y, Tsuda T, Takikawa T, Yoshida T, Ohashi K, Shimizu Y, et al: Cardiomyocytes capture stem cell-derived, anti-apoptotic microRNA-214 via clathrin-mediated endocytosis in acute myocardial infarction. J Biol Chem 294: 11665-11674, 2019.

46. Sun R, Liu Z, Han L, Yang Y, Wu F, Jiang Q, Zhang H, Ma R, Miao J, He K, et al: miR-22 and miR-214 targeting BCL9L inhibit proliferation, metastasis, and epithelial-mesenchymal transition by down-regulating Wnt signaling in colon cancer. FASEB J 33: 5411-5424, 2019.

47. Okazaki Y, Chew SH, Nagai H, Yamashita Y, Ohara H, Jiang L, Akatsuka S, Takahashi T and Toyokuni S: Overexpression of miR-199/214 is a distinctive feature of iron- and asbestos-induced sarcomatoid mesothelioma in rats. Cancer Sci 2020 (Epub ahead of print).

48. Kong L, Liu P, Zheng M, Wang Z, Gao Y, Liang K, Wang H and Tan X: The miR-1224-5p/ELF3 axis regulates malignant behaviors of pancreatic cancer via PI3K/AKT/Notch signaling pathways. Onco Targets Ther 13: 3449-3466, 2020.

49. Duan Y, Tan Z, Yang M, Li J, Liu C, Wang C, Zhang F, Jin Y, Wang Y and Zhu L: PC-3-derived exosomes inhibit osteoclast differentiation by downregulating miR-214 and blocking NF- $\kappa \mathrm{B}$ signaling pathway. Biomed Res Int 2019: 8650846, 2019.

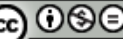

This work is licensed under a Creative Commons Attribution-NonCommercial-NoDerivatives 4.0 International (CC BY-NC-ND 4.0) License. 